\title{
Optical data storage by using orthogonal wavelength-multiplexed volume holograms
}

\author{
George A. Rakuljic and Victor Leyva
}

Accuwave Corporation, 1653 19th Street, Santa Monica, California 90404

Amnon Yariv

California Institute of Technology, Pasadena, California 91125

Received May 19, 1992

\begin{abstract}
We propose a volume holographic data storage scheme that employs counterpropagating reference and image beams and wavelength multiplexing for page differentation. This method is compared with that based on angular multiplexing. A reduction in holographic cross talk is predicted. Further cross-talk reduction that is due to sidelobe suppression is observed in experiments by using photorefractive crystals and the proposed orthogonal data storage.
\end{abstract}

Optical data storage in volume holograms has been an exciting prospect since the early days of holography. ${ }^{1}$ This is due mostly to the theoretical storage capacity of $\sim V / \lambda^{3}$ bits in a volume $V$. This, as an example, translates to $8 \times 10^{12}$ bits in a $1-\mathrm{cm}^{3}$ volume at $\lambda=0.5 \mu \mathrm{m}$.

Early attempts at exploiting this potential have been disappointing. ${ }^{2-10}$ Even though hundreds of holograms have been successfully stored in a single volume in these experiments, the data content of the individual holograms was minimal, and, therefore, so was the overall volumetric information density. This was due partly to material limitations ${ }^{11,12}$ but, more fundamentally, to the intolerably high cross talk resulting from the angular multiplexing method used to record the large number of holograms. ${ }^{4-7}$ In this traditional method, many twodimensional images are recorded sequentially, each with its unique angularly multiplexed reference wave. Recently, however, phase-encoded or spatially orthogonal reference beams have been used to multiplex many holograms..$^{13,14}$ These approaches, nonetheless, are variations of the basic angular multiplexing technique and differ fundamentally from the orthogonal wavelength-multiplexed storage technique proposed here.

To appreciate the nature of the problem we recall that the basic property, and virtue, of holography is the essentially uniform distribution of the recorded information throughout the physical volume of the recording medium. We assert here the self-evident, but heretofore ignored, fact that it is equally important to distribute the information throughout the grating $\mathbf{K}$ space as well. Failure to do so increases the cross talk between the reconstructed holograms.

Consider a sinusoidal volume grating written by two plane waves $\mathbf{E}_{1} \exp \left\{i\left[\omega t-\mathbf{k}^{(1)} \cdot \mathbf{r}\right]\right\}$ and $\mathbf{E}_{0} \exp \left\{i\left[\omega t-\mathbf{k}^{(0)} \cdot \mathbf{r}\right]\right\}$ described by the index-ofrefraction distribution

$$
\Delta n \propto\left|E_{1} E_{0}\right| \sin \left(\mathbf{K} \cdot \mathbf{r}+\phi_{1}-\phi_{0}\right),
$$

where

$$
\mathbf{K}=\mathbf{k}^{(1)}-\mathbf{k}^{(0)}, \quad E_{1,0}=\left|\mathbf{E}_{1,0}\right| \exp \left(i \phi_{1,0}\right) .
$$

Such a grating can be identified by the point $\mathbf{K}$ in $K_{x}, K_{y}, K_{z}$ space. If the wave $\mathbf{E}_{0}$ is spatially modulated, say by image information, the corresponding $\mathbf{K}$ point becomes a surface whose extent (or solid angle) is determined by the smallest feature size of the information. Such a surface can be labeled by the reference wave used to record it and is shown as $\mathbf{K}_{\text {grating }}^{(1)}$ in Fig. 1 along with the responsible $\mathbf{k}_{\text {ref }}^{(1)}$. The same figure also shows the surface $\mathbf{K}_{\text {grating }}^{(2)}$ that is due to a second image recorded with a second, angle-multiplexed reference wave $\mathbf{k}_{\mathrm{ref}}^{(2)}$.

Reconstruction of, say, image 2 by illuminating the stored holograms with a wave along $\mathbf{k}_{\mathrm{ref}}^{(2)}$ will inevitably lead to undesirable parasitic scattering

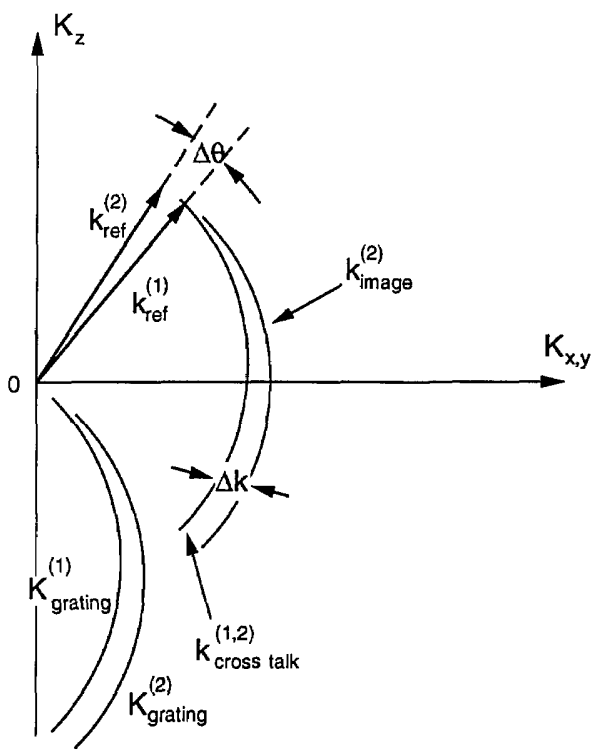

Fig. 1. $\quad \mathbf{K}$ space diagram for volume holography that uses angle-multiplexed storage. 


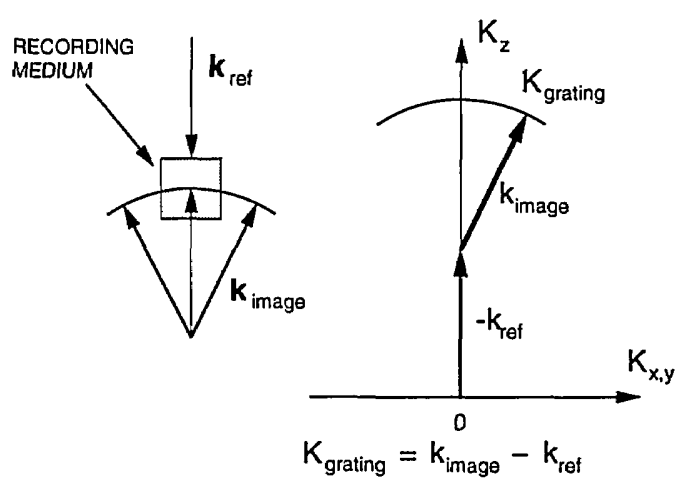

(a)

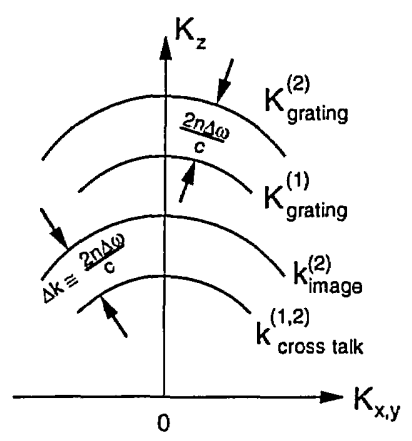

(b)

Fig. 2. K space diagrams for volume holography that uses orthogonal data storage: (a) single hologram; (b) multiple hologram storage.

of the incident wave off the recorded hologram of image 1. The locus of the desired reconstructed $\mathbf{k}_{\text {image }}^{(2)}$ vectors in this case are shown in the figure as well as those of the undesired (cross talk) $\mathbf{k}_{\text {cross talk vec- }}^{(1,2)}$ tors due to the scattering off grating 1 by reference beam 2. A relative measure of the cross talk is ${ }^{15}$

$$
\eta_{\text {cross talk }} \approx \frac{\kappa^{2}}{\kappa^{2}+(\Delta k)^{2}},
$$

where $\kappa$ is the coupling constant of the grating and $\Delta k$ is the spacing in $\mathbf{K}$ space. In regions of $\mathbf{K}$ space where the separation $\Delta k$ between $\mathbf{k}_{\text {image }}^{(2)}$ and $\mathbf{k}_{\text {cross talk }}^{(1,2)}$ is small, the cross talk between holograms 1 and 2 approaches $100 \%$. From Fig. 1 it is evident that such regions exist and that the problem is exacerbated by increasing the information content of the beams, and thus the spread in $\mathbf{K}$ space. It is therefore imperative to distribute the gratings as uniformly as possible in $\mathbf{K}$ space so as to keep the spacing $\Delta k$ at a nearly uniform and as large a value as possible, consistent with a given information content of the recorded images.

One method that distributes information uniformly in $\mathbf{K}$ space is shown in Fig. 2. Holograms are recorded by counterpropagating image and reference waves $\mathbf{k}_{\text {image }}$ and $\mathbf{k}_{\text {ref }}$, respectively, as in the figure. All the reference waves propagate along the same, say, $z$ axis, but each is of a slightly different wavelength. The $\mathbf{K}_{\text {grating }}$ surface of a single picture is shown in Fig. 2(a).

Figure 2(b) shows what happens when we record two images using two beams differing in frequency by $\Delta \omega / 2 \pi$. The increase in the information content of a given image causes a mostly lateral spread of the loci, which allows a more uniform coverage of $\mathbf{K}$ space. The mismatch parameter $\Delta k$ that determines the relative intensity of the cross talk is nearly constant in magnitude so that the level of cross talk is essentially independent of the information content (spatial detail) of the images. This indicates the potential for high data storage capacity by using the proposed storage scheme. The orthogonal nature of the curves in Fig. 2 has led us to call this scheme orthogonal data storage. ${ }^{16,17}$

Experiments were conducted with a 2-mm-thick $z$-cut and polished lithium niobate $\left(\mathrm{LiNbO}_{3}\right)$ crystal. Figure 3 shows the wavelength dependence of the diffraction efficiency in the reflection geometry. The hologram was recorded with $\lambda=641.7 \mathrm{~nm}$ and the geometry of Fig. 2. The figure also shows a theoretical plot of the expected diffraction efficiency. One major surprise is the smaller cross talk of the actual grating compared with the theoretically predicted values. As an example, at $1 \mathrm{~nm}$ from the recording wavelength of $641.7 \mathrm{~nm}$, the measured value is $-44 \mathrm{~dB}$ of the peak value compared with a theoretical value of $-37 \mathrm{~dB}$. We believe that the discrepancy is due to an effective apodization whose origin is not fully understood.

The holographic cross talk in an orthogonal data storage scheme was investigated by recording two different, high-resolution holograms written with wavelengths $2 \mathrm{~nm}$ apart. Integrated circuit masks consisting of $1-5-\mu \mathrm{m}$-wide lines were used as the transparency $\mathrm{T}$ in the experimental arrangement of Fig. 4. The writing beams were oriented in the counterpropagating geometry of orthogonal data storage. Figure 5 summarizes the experimental results. A holographic cross-talk level of $-43 \mathrm{~dB}$ is measured at the vacant address at $642 \mathrm{~nm}$, centered $1 \mathrm{~nm}$ between the other two informationladen holograms. This value is essentially the same as the plane-wave (no information) result of Fig. 3, which thus shows that holographic cross talk does not increase in orthogonal data storage as substantial amounts of information are added to the images. Since $\Delta k$ at a given level of cross talk scales as the inverse of the hologram length, the data of Fig. 5 are consistent with a cross-talk level of $<-40 \mathrm{~dB}$ with

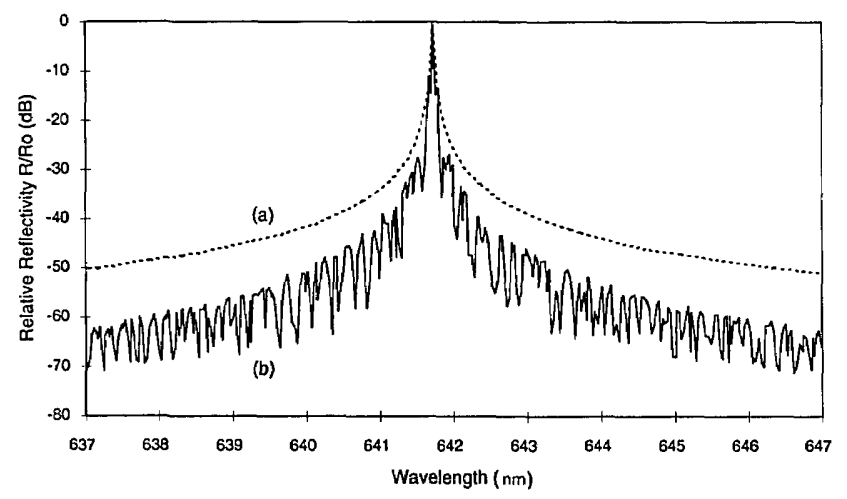

Fig. 3. Relative spectral responses $\left[10 \log _{10}\left(R / R_{0}\right)\right]$ of 2-mm-thick planar holograms: curve (a), theoretical (envelope only); curve (b), experimental. 


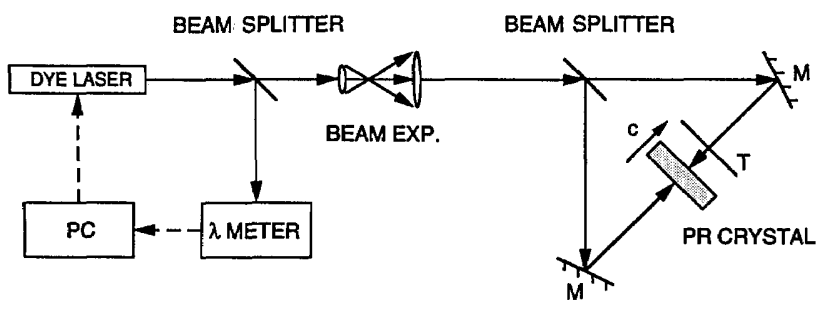

Fig. 4. Experimental setup for holographic recording with orthogonal data storage. $\mathrm{PC}$, personal computer; M's, mirrors.

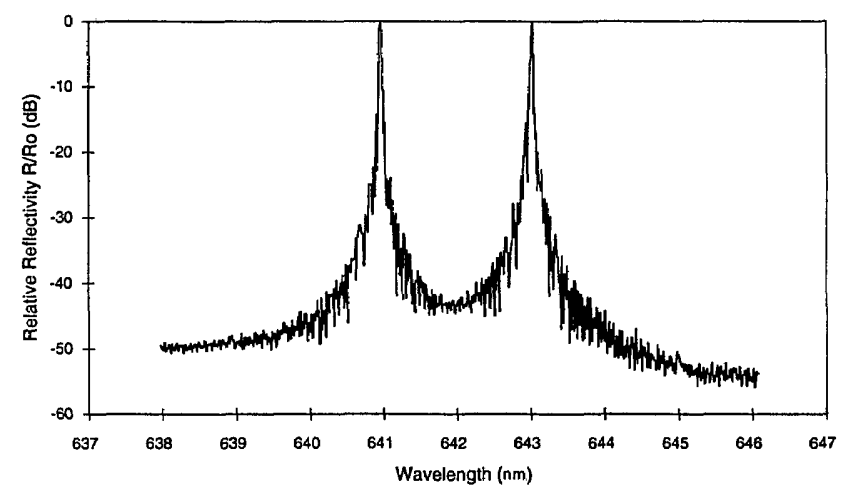

Fig. 5. Relative spectral response $\left[10 \log _{10}\left(R / R_{0}\right)\right]$ of two high-resolution ( $\sim 1-\mu \mathrm{m}$ features) images recorded holographically in a 2-mm-thick photorefractive crystal by using orthogonal data storage.

holograms written every $\Delta \lambda \approx 0.1 \mathrm{~nm}$ in a crystal $1 \mathrm{~cm}$ long.

In summary, the orthogonal data storage approach to volume holography is described. It is based on recording holograms between counterpropagating image and reference waves with a single propagation axis. Successive holograms are recorded and reconstructed by using wavelength multiplexing. The holographic cross talk between wavelength channels is shown to be less than that obtained with conventional angular multiplexing. With orthogonal data storage the cross talk between holograms does not increase with added spatial information, unlike the case of angular multiplexing. In fact, it remains at essentially the plane-wave hologram value. Further cross-talk reduction is observed by using photorefractive crystals as the recording media and orthogonal data storage due to sidelobe suppression effects.

We thank Bob D. Guenther of the U.S. Army Research Office for helpful discussions about holographic data storage.

\section{References}

1. P. J. van Heerden, Appl. Opt. 2, 393 (1963).

2. L. d'Auria, J. P. Huignard, C. Slezak, and E. Spitz, Appl. Opt. 13, 808 (1974).

3. L. Solymar and D. J. Cooke, Volume Holography and Volume Gratings (Academic, London, 1981).

4. W. J. Burke, "Signal/noise ratio of holographic images," Final Report, contract no. N00019-75-M-0494 (RCA Laboratories, Princeton, N.J., October 1975).

5. W. J. Burke, P. Sheng, and H. A. Weakliem, "Intrinsic noise sources in volume holography," Final Report, contract no. N00014-75-C-0590 (RCA Laboratories, Princeton, N.J., December 1975).

6. W. J. Burke and P. Seng, J. Appl. Phys. 48, 481 (1977).

7. K. Blotekjaer, Appl. Opt. 18, 57 (1979).

8. L. Hesselink and S. Redfield, Opt. Lett. 13, 877 (1988).

9. P. D. Henshaw, S. A. Lis, and N. R. Guivens, Jr., "Compact 4-D optical neural network architecture," Final Report, contract no. F49620-89-C-0120 (Sparta, Inc., Lexington, Mass., April 1990).

10. F. H. Mok, M. C. Tackitt, and H. M. Stoll, Opt. Lett. 16, 605 (1991).

11. A. M. Glass, Opt. Eng. 17, 470 (1978).

12. P. Gunter, Phys. Rep. 93, 199 (1982).

13. C. Denz, G. Pauliat, G. Roosen, and T. Tschudi, in Digest of Meeting on Photorefractive Materials, Effects, and Devices (Optical Society of America, Washington, D.C., 1991), paper MD-1.

14. Y. Taketomi, J. Ford, H. Sasaki, J. Ma, Y. Fainman, and S. H. Lee, in Digest of Meeting on Photorefractive Materials, Effects, and Devices (Optical Society of America, Washington, D.C., 1991), paper MD-2.

15. A. Yariv, Optical Electronics, 3rd ed. (CBS College Publishing, New York, 1985), Eq. 13.8-7, p. 438.

16. G. A. Rakuljic, A. Yariv, and V. Leyva, in Digest of Meeting on Photorefractive Materials, Effects, and Devices (Optical Society of America, Washington, D.C., 1991), paper MD-3.

17. V. Leyva, G. A. Rakuljic, and A. Yariv, in Digest of Optical Society of America Annual Meeting (Optical Society of America, Washington, D.C., 1991), paper FU-7. 